\title{
CRISPR-on for activation of endogenous SMARCA4 and TFAP2C expression in bovine embryos
}

\author{
Virginia Savy¹, Virgilia Alberio¹, Natalia G Canel ${ }^{1}$, Laura D Ratner', Maria I Gismondi², \\ Sergio F Ferraris ${ }^{3}$, Rafael Fernandez-Martín¹, Jason G Knott ${ }^{4}$, Romina J Bevacqua ${ }^{1,5}$ and \\ Daniel F Salamone ${ }^{1}$ \\ ${ }^{1}$ Laboratorio de Biotecnología Animal, Facultad de Agronomía, Universidad de Buenos Aires/INPA CONICET, \\ CABA, Argentina, ${ }^{2}$ Instituto de Biotecnología, CICVYA, INTA, Hurlingam, Buenos Aires, Argentina, ${ }^{3}$ Centro de \\ Ciencias Veterinarias, Universidad Maimónides, CABA, Argentina, ${ }^{4}$ Department of Animal Science, Michigan State \\ University, East Lansing, Michigan, USA and ${ }^{5}$ Seung Kim Lab, Department of Developmental Biology, Stanford \\ University School of Medicine, Stanford, California, USA \\ Correspondence should be addressed to D F Salamone; Email: Salamone@agro.uba.ar
}

(R J Bevacqua and D F Salamone contributed equally to this research)

\begin{abstract}
CRISPR-mediated transcriptional activation, also known as CRISPR-on, has proven efficient for activation of individual or multiple endogenous gene expression in cultured cells from several species. However, the potential of CRISPR-on technology in preimplantation mammalian embryos remains to be explored. Here, we report for the first time the successful modulation of endogenous gene expression in bovine embryos by using the CRISPR-on system. As a proof of principle, we targeted the promoter region of either SMARCA4 or TFAP2C genes, transcription factors implicated in trophoblast lineage commitment during embryo development. We demonstrate that CRISPR-on provides temporal control of endogenous gene expression in bovine embryos, by simple cytoplasmic injection of CRISPR RNA components into one cell embryos. dCas9VP160 activator was efficiently delivered and accurately translated into protein, being detected in the nucleus of all microinjected blastomeres. Our approach resulted in the activation of SMARCA expression shortly after microinjection, with a consequent effect on downstream differentiation promoting factors, such as TFAP2C and CDX2. Although targeting of TFAP2C gene did not result in a significant increase in TFAP2C expression, there was a profound induction in CDX2 expression on day 2 of development. Finally, we demonstrate that CRISPR-on system is suitable for gene expression modulation during the preimplantation period, since no detrimental effect was observed on microinjected embryo development. This study constitutes a first step toward the application of the CRISPR-on system for the study of early embryo cell fate decisions in cattle and other mammalian embryos, as well as to design novel strategies that may lead to an improved trophectoderm development.

Reproduction (2020) $\mathbf{1 5 9} 767-778$
\end{abstract}

\section{Introduction}

Over the past 10 years, engineered-programmable DNA nucleases have resulted in unprecedented opportunities for gene editing. The CRISPR/Cas9 system (from Clustered Regularly Interspaced Short Palindromic Repeat/CRISPR-associated nuclease9) stands out among engineered-nucleases given its simple design, flexibility and species adaptability (Mojica et al. 2005, Jinek et al. 2012, Cong et al. 2013, Knott \& Doudna 2018). This system consists of a single guide RNA (sgRNA), which directs the nuclease Cas9 to its target sequence in the genome by complementary base pairing, generating double-stranded breaks that, upon repair by either nonhomologous end-joining or homologous recombination, can potentially result in the knock-out of any gene of interest.

Soon after its introduction as gene editing tool, the CRISPR/Cas9 system was also re-purposed for multiple site-specific nuclease-independent modifications (Larson et al. 2013, Zalatan et al. 2015, Jusiak et al. 2016, Knott \& Doudna 2018, Engreitz et al. 2019). To achieve this, the Cas9 nuclease catalytic domains were mutated, resulting in a DNA-binding protein still capable of site-specific target recognition but unable to cut the DNA, called dCas9 (for dead Cas9, Qi et al. 2013). The fusion of dCas9 to different effector domains, including transcriptional activation or repression domains, allowed specific modulation of gene expression in vitro (Gilbert et al. 2013, Mali et al. 2013, Maeder et al. 2013, Perez- 
Pinera et al. 2013). The first version of CRISPR-based transcriptional activator or CRISPR-on, consisted on the fusion of the dCas9 protein to several tandem repeats of VP16 herpes virus activation domain to generate the dCas9VP64 programmable activator (Gilbert et al. 2013, Perez-Pinera et al. 2013). Subsequent 'secondgeneration' activators were developed in order to co-recruit multiple activators by using the dCas9 protein as a scaffold. CRISPR-on tools have been successfully used to turn on the expression of individual or multiple endogenous genes in cell culture from several species (Cheng et al. 2013, Gilbert et al. 2013, Maeder et al. 2013, Perez-Pinera et al. 2013, Konermann et al. 2015). However, up to date, CRISPR-on-mediated endogenous gene activation has not been applied to mammalian embryos.

The proper development of the early mammalian embryo involves both temporal and spatial regulation of gene expression. During early embryogenesis, the first cell fate decision results in segregation of the inner cell mass (ICM), which will ultimately contribute mainly to the fetus, and trophectoderm (TE), which serves as the precursor of extraembryonic tissues. This early differentiation occurs before implantation and has been widely studied in mice. Particularly in this species, lineage segregation is directed by the antagonistic expression of Oct4 and $C d \times 2$ followed by OCT4-specific localization to the pluripotent ICM and CDX2 confinement to the trophectoderm epithelium at the late blastocyst stage (Rossant 2004). The pluripotent state of the murine ICM is maintained through the formation of a complex between OCT4, SOX2 and NANOG (Avilion et al. 2003, Masui et al. 2007). Conversely, CDX2 plays a key role in trophoblast specification through the repression of Oct4 and Nanog expression (Strumpf et al. 2005, Chen et al. 2009).

Although early embryo development is highly conserved within mammalian species, there are several species-specific differences underlying lineage commitment. As reported for mouse, the OCT4/SOX2/ NANOG protein complex is also present in the bovine ICM (Degrelle et al. 2005, Ozawa et al. 2012) and CDX2 is located exclusively in theTE at the late blastocysts stage. However, while CDX2 is essential for TE maintenance, it is not crucial for TE establishment as it is for mouse (Berg et al. 2011). Moreover, in bovine, as well as in humans and pigs, the mechanism of differentiation does not require the restriction of OCT4 to the blastocyst ICM. In fact, OCT4 and CDX2 transcripts are co-expressed in the TE of in vitro-produced bovine embryos on day 7 (Simmet et al. 2018). It is only by day 11 that OCT4 expression is repressed in the TE lineage (Berg et al. 2011) suggesting that, in cattle, TE commitment might be regulated upstream of CDX2 and that cell fate segregation mechanisms have diverged between cattle and mouse.
Recently, two transcription factors (TF), TFAP2C and SMARCA4, were shown to have key roles in trophoblast lineage commitment through the modulation of CDX2 and NANOG expression in murine embryos (Cao et al. 2015, Carey et al. 2015). TFAP2C is a TF with a high degree of sequence conservation present in a wide variety of species, playing an important role in the placenta development (Aston et al. 2009, Lee et al. 2015). In mice, TFAP2C mRNA abundance remains stable through fertilization to the blastocysts stage (Winger et al. 2006) and promotes differentiation toward the TE by regulating the establishment of cell polarity, repressing positiondependent HIPPO signaling, and activating $C d x 2$ expression (Cao et al. 2015). SMARCA4 (also known as BRG1) is a key component of a remodeling chromatin protein complex that mediates crucial developmental events such as embryonic genome activation (EGA) and cell differentiation, through both activation and repression of gene expression. In mouse, it has been reported that Oct4, Nanog and Sox2 are direct targets of SMARCA4 and Smarca4 lack of expression impairs embryo implantation (Kidder et al. 2009, Choi et al. 2013, Carey et al. 2015). Since, in cattle, TE confinement seems to be regulated upstream of CDX2, it is likely that in this species SMARCA4 and TFAP2C have a more crucial role in the initial lineage segregation toward ICM and TE.

Based on the above, in this work we applied for the first time the CRISPR-on system to bovine preimplantation embryos for modulation of endogenous expression of SMARCA4 and TFAP2C. Placentation failures are frequently reported for bovine in vitro embryo production (Hill et al. 2000, Chavatte-Palmer et al. 2012) and may be associated with aberrant trophoblast cell proliferation and differentiation during the pre-implantation stage (Knott \& Paul 2014, Mainigi et al. 2016). Thus, we hypothesized that CRISPR-on may be suitable to induce an activation of SMARCA4 and/or TFAP2C expression which ultimately would promote cell differentiation toward the TE lineage. To achieve this, in an initial experiment, we injected early zygotes with dCas9VP160 mRNA and evaluated dCas9VP160 mRNA stability and translation into protein during embryo development. Subsequently, we employed the CRISPR-on technology for activation of endogenous SMARCA4 and TFAP2C, by simple cytoplasmic injection of dCas9VP160 together with the appropriate sgRNAs. Finally, we assessed the effect of transcriptional activation on preimplantation embryo development and TE proportion at the blastocyst stage. The possibility to precisely modulate the transcription of endogenous genes in a simple and transient fashion opens new and unprecedented possibilities to the study of cell differentiation in species other than mice and also to easily be applied for bovine in vitro embryo production programs improvement, since modulation of endogenous gene expression with CRISPR-on is independent of gene editing. 


\section{Materials and methods}

\section{Experimental design}

The experiments described below were conducted to evaluate the potential of CRISPR-on system for modulation of endogenous gene expression in preimplantation bovine embryos:

In an initial experiment, dCas9VP160 mRNA decay and translation were assessed during embryo development. To do this, presumptive zygotes were cytoplasmically injected with dCas9VP160 mRNA and mRNA relative abundance was evaluated 2, 4 and 7 days after microinjection by RT-qPCR. The immunodetection of dCas9VP160 protein in bovine microinjected embryos was also assessed. In a second experiment, CRISPR-mediated activation of SMARCA4 and TFAP2C expression was evaluated. The general scheme of the procedure is shown on Fig. 1A. First, four non-overlapping sgRNA were designed and synthesized targeting either bovine SMARCA4 or TFAP2C proximal promoter region (Fig. 1B). Presumptive zygotes were microinjected with the dCas9VP160 mRNA and appropriated pool of sgRNAs, Table 1 summarizes the groups and microinjection components used. Embryos were in vitro cultured until day 2, 4 or 7 and stored for RT-qPCR analysis, as indicated in Fig. 1. RT-qPCR assay was performed to evaluate the expression of the targeted genes and downstream genes. Finally, in a third experiment, the effect of transcriptional modulation over embryo development was assessed by comparing cleavage, morulae and blastocysts rates within groups, at days 2, 5 and 7 of the in vitro culture.

\section{Oocyte collection and in vitro maturation}

Bovine ovaries were obtained from local slaughterhouses and transported to the laboratory at $25-30^{\circ} \mathrm{C}$. Cumulus oocyte complexes (COCs) were aspirated from 2 to $8 \mathrm{~mm}$ follicles and collected into TALP-H medium. COCs were cultured for $24 \mathrm{~h}$ in $100 \mu \mathrm{L}$ droplets of complete TCM medium, under mineral oil in $6.5 \% \mathrm{CO}_{2}$ in humidified air at $39^{\circ} \mathrm{C}$, for in vitro maturation. Complete TCM medium consisted of M-199
(31100-035; Thermo Fisher Scientific), supplemented with 10\% FCS (013/07; Internegocios, Mercedes, Buenos Aires, Argentina), $10 \mathrm{mg} / \mathrm{mL}$ follicle-stimulating hormone (NIHFSH-P1, FolltropinR, Bioniche, Caufield North, Victoria, Australia), $0.3 \mathrm{mM}$ sodium pyruvate (P2256), $100 \mathrm{mM}$ cysteamine (M9768), and 2\% antibiotic-antimycotic (ATB, 15240-096; Thermo Fisher Scientific).

\section{In vitro fertilization (IVF) procedure}

Frozen sperm straws of bulls with proven fertility were thaw in a $37^{\circ} \mathrm{C}$ water bath and in Brackett-Oliphant (BO) medium (Brackett \& Oliphant 1975). Spermatozoa were then washed twice by centrifugation at $490 \mathrm{~g}$ for $5 \mathrm{~min}$ to remove cryoprotectants and finally resuspended in $\mathrm{BO}$ supplemented with $5 \mathrm{mM}$ caffeine (C4144, SIGMA) and 20 IU/mL heparin (H3149, SIGMA). Spermatozoa were adjusted to $16 \times 10^{6} / \mathrm{mL}$ with BO containing fatty acid-free BSA $(10 \mathrm{mg} /$ $\mathrm{mL}$, A6003 SIGMA). For IVF, 20 to 25 COCs were placed in $100 \mu \mathrm{L}$ droplets of sperm suspension for $5 \mathrm{~h}$ under $5 \% \mathrm{CO} 2$ in humidified air. Then, cumulus cells were removed by vortexing the presumptive zygotes for $2 \mathrm{~min}$ in hyaluronidase $(\mathrm{H}-4272)(1 \mathrm{mg} / \mathrm{mL}$ in Dulbecco's PBS) and then washed 3 times in TALP-Hepes. Only presumptive zygotes were used for microinjection.

\section{CRISPR-on system}

The Zhang Lab CRISPR design tool (http://crispr.mit.edu) was used to design four non-overlapping sgRNAs, targeting the proximal promoter region of either SMARCA4 or TFAP2C. For both genes, the regulatory region ranging from -200 to $-50 \mathrm{bp}$ upstream of the TSS was considered as the optimal activation window (Fig. 1B, Gilbert et al. 2013, Konermann et al. 2015). In all cases, the design parameters were set to minimize off-target binding sites in the genome. pUC57-sgRNA expression vector was a gift from Xingxu Huang (Addgene plasmid \#51132; http://n2t.net/addgene:51132; Shen et al. 2014). All sgRNAs were cloned into pUC57-sgRNA

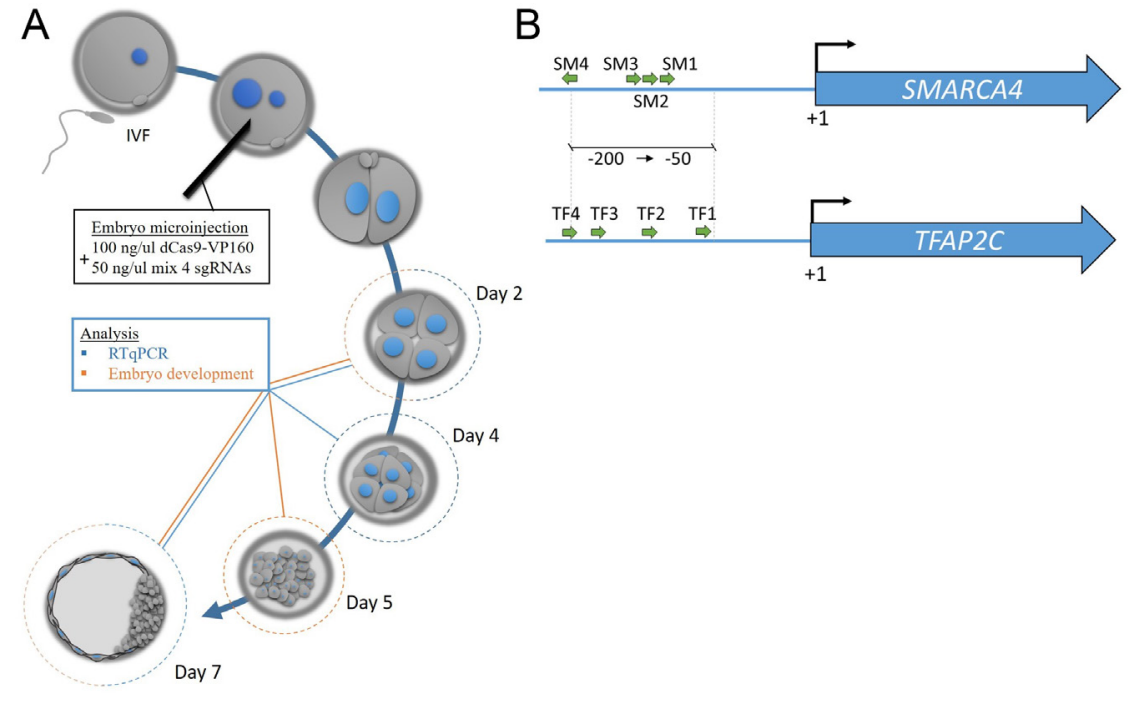

Figure 1 (A) Scheme of general procedure for CRISPR-mediated modulation of gene expression in bovine embryos. Gene expression was assessed by RT-qPCR from pools of 10 embryos at day 2 or day 4 of the in vitro culture or 5 blastocysts at day 7 . Data sets were analyzed by Mann-Whitney $t$-test. Embryo development was assessed by cleavage, morulae and blastocysts rates at days 2, 5 and 7 of the in vitro culture, respectively. Differences were determined by Fisher's exact test. (B) Scheme of B. taurus SMARCA4 and TFAP2 $C$ regulatory region. Green arrows indicate orientation and position of 4 non-overlapping sgRNAs designed for transcriptional activation of targeted genes. The optimal activation window was considered between -200 and -50 bp upstream of each TSS, indicated in the figure as ' +1 '. 
Table 1 Experimental groups and components of microinjection mix.

\begin{tabular}{lccc}
\hline & dCas9_SM & dCas9_TF & SHAM \\
\hline dCas9VP160 & + & + & + \\
SM1, SM2, SM3, SM4 & + & - & - \\
TF1, TF2, TF3, TF4 & - & + & - \\
\hline
\end{tabular}

SM (1, 2, 3 and 4) are sgRNAs targeting SMARCA4 promoter region. TF (1, 2, 3 and 4) are sgRNAs targeting TFAP2C promoter region.

Microinjection mix was prepared in PVP.

expression vector and the correct sequence was confirmed by capillary Sanger sequencing (Genomic Unit, Biotechnology Institute, INTA, Hurlingham, Argentina). dCas9 fused to 10 tandems repeats of VP16 domain (dCas9VP160) was used as a programmable transcriptional activator. Plasmid coding for dCas9VP160 was a gift from Rudolf Jaenisch (Addgene plasmid \#48240; http://n2t.net/addgene:48240; Cheng et al. 2013). In order to generate dCas9VP160 mRNA, a pT3dCas9VP160 PCR product was amplified using a Forward primer containing the T3 sequence (T3_dCas9VP160_f) and a BGH-Reverse primer $\left(1\right.$ cycle at $95^{\circ} \mathrm{C}$ for $5 \mathrm{~min}$, followed by $95^{\circ} \mathrm{C}$ for $30 \mathrm{~s}, 58^{\circ} \mathrm{C}$ for $30 \mathrm{~s}$ and $68^{\circ} \mathrm{C}$ for $5 \mathrm{~min}$, during 35 cycles, and a final extension at $68^{\circ} \mathrm{C}$ for $\left.5 \mathrm{~min}\right)$. The Q5 High fidelity DNA polymerase (M0491s, NEB) was employed for accurate amplification. The PCR product was purified using the QIAquick PCR Purification Kit (Qiagen, 28104) and used as template for in vitro transcription (IVT) reaction. The sequences of all sgRNAs and primers used for dCas9VP160 amplification are listed in Table 2 .

\section{In vitro transcription (IVT) of RNAs and injection of bovine zygotes}

For sgRNAs IVT, the MEGAshortscript Kit (AM1354, Ambion) was used, with Dral-digested pUC57-sgRNA plasmids as templates. sgRNAs were purified using the MEGAclear Kit (AM1908, Ambion). For dCas9VP160 IVT, the pT3dCas9VP160 PCR product was used as template. Capped mRNA was synthesized using mMESSAGE mMACHINETM T3 Transcription Kit (Ambion, AM1348) and purified with the RNeasy Mini Kit (Qiagen). dCas9VP160 mRNA proper size was confirmed by denaturing gel electrophoresis. dCas9VP160 mRNA (100 ng/ $\mu \mathrm{L})$ and mix of 4 sgRNAs targeting either SMARCA4 or TFAP2C regulatory region $(25 \mathrm{ng} / \mu \mathrm{L}$ for each) were mixed in PVP and microinjected into the cytoplasm of bovine presumptive zygotes.

\section{In vitro embryo culture}

Presumptive zygotes were cultured in synthetic oviductal fluid medium (SOF, Holm et al. 1999) supplemented with $2.5 \%$ FBS at $39^{\circ} \mathrm{C}$ in humidified gas mixture $\left(5 \% \mathrm{CO}_{2}, 5 \% \mathrm{O}_{2}, 90 \%\right.$ $\mathrm{N}_{2}$ ). Culture medium was renewed on days 2 and 5 of culture. Cleavage was evaluated on day 2 and morulae and blastocyst rate were determined on day 5 and 7 , respectively.

\section{RNA isolation and RT-qPCR analysis}

Pools of ten day 2 or day 4 embryos or pools of 5 blastocysts were stored at $-20^{\circ} \mathrm{C}$ in RNA later (AM7020, Ambion) until RNA extraction. The Arcturus PicoPure RNA isolation kit (Applied Biosystems) was used for total RNA isolation, according to manufacturer's instruction and eluted in $11 \mu \mathrm{L}$. First-strand cDNA was synthesized using random primers and the SuperScript II reverse transcriptase (Life Technologies) with $10 \mu \mathrm{L}$ of total RNA in a $20-\mu \mathrm{L}$ final reaction. qPCR analysis was performed with gene-specific primers (Table 3), using SYBR green detection on a StepOne Plus thermocycler (Applied Biosystems). Primers targeting SMARCA4 promoter were designed from mRNA and genomic sequences obtained from NCBI, using PerlPrimer ${ }^{\circledR}$ Software v1.1.21 (Marshall 2004). At least three biological replicates of IVF were evaluated for all time points assessed, each one including non-injected as well as injected groups. Samples were loaded as duplicates (technical replicates) in a $15 \mu \mathrm{L}$ reaction, containing $8 \mu \mathrm{L}$ Sybr green, $1 \mu \mathrm{L}$ of each primer $(20 \mathrm{mmol} / \mu \mathrm{L}), 1 \mu \mathrm{L}$ cDNA template, and $4 \mu \mathrm{L}$ Milliq water. Data was analyzed by the $\Delta \Delta \mathrm{CT}$ method using the geometric mean of ACTIN and GAPDH as internal standard. One biologica.

\section{Immunocytochemistry (ICC)}

Preimplantation stage embryos were analyzed by immunofluorescence to determine dCas9VP160 protein presence 3 days after microinjection. Embryos were fixed in $4 \%$ Paraformaldehyde (F-1635, Sigma) in DPBS (14287072; Gibco) for $20 \mathrm{~min}$ and permeabilized in $0.1 \%$ Triton-X

Table 2 Sequence of primers used to obtain the dCas9VP160 coding sequence under the T3 promoter and sgRNAs used to target SMARCA4 and TFAP2C proximal promoter regions.

\begin{tabular}{lll}
\hline Abrev & Name & Sequence $\left(5^{\prime}-3^{\prime}\right)$ \\
\hline SM1 & sgRNA_SMARCA4_1Fw & TAGGGCGGGGGCGCGGGCAGCGTG \\
SM2 & sgRNA_SMARCA4_2Fw & TAGGGAAGCGAGAGAGGGAGTTCG \\
SM3 & sgRNA_SMARCA4_3Fw & TAGGGCACGCGCGCTAGGAGCGGA \\
SM4 & sgRNA_SMARCA4_4Fw & TAGGGTTGTCTGGGAGAGGTGGGT \\
TF1 & sgRNA_TFAP2C_1Fw & TAGGGTCCTTCTCATTAAGGCTCCC \\
TF2 & sgRNA_TFAP2C_2Fw & TAGGGCTAGGCTGGGACTGGCTGG \\
TF3 & sgRNA_TFAP2C_3Fw & TAGGGCGGAGACCCACGCTGCGTG \\
TF4 & sgRNA_TFAP2C_4Fw & TAGGGTGCGTGGTGAGGGTGACTGA \\
& T3_dCas9VP160_F & AATTAACCCTCACTAAAGGGAGAcaggttggaccggtgccaccA \\
& BGH-Rev & TAGAAGGCACAGTCGAGG \\
\hline
\end{tabular}

a Uppercase letters indicate the T3 minimum promoter sequence. 
Table 3 Sequence of primers used for RT-qPCR analysis in bovine embryos.

\begin{tabular}{|c|c|c|c|c|c|}
\hline \multirow[b]{2}{*}{ Gene } & \multirow[b]{2}{*}{ Accession number } & \multicolumn{2}{|c|}{ Primer Sequence $\left(5^{\prime}-3^{\prime}\right)$} & \multirow[b]{2}{*}{ Amplicon (bp) } & \multirow[b]{2}{*}{ Reference } \\
\hline & & Forward & Reverse & & \\
\hline TFAP2C & NM_001083748 & ATTCGCAAAGGTCCTATTTCCA & TAGATGTAGAGCTGAGGAGGGA & 139 & $\begin{array}{l}\text { Zhenhua et al. } \\
2017\end{array}$ \\
\hline$C D \times 2$ & AM293662 & CGTCTGGAGCTGGAGAAGGA & CGGCCAGTTCGGCTTTC & 70 & $\begin{array}{l}\text { Tripurani et al. } \\
2011\end{array}$ \\
\hline NANOG & - & TCCAGCAAATGCAAGAACTTTC & TTACATTTCATTCTCTGGTTCTGGAA & 88 & $\begin{array}{l}\text { Wrenzycki et al. } \\
2002\end{array}$ \\
\hline GAPDH & NM_001034034 & TTCAACGGCACAGTCAAGG & ACATACTCAGCACCAGCATCAC & 119 & Khan et al. 2012 \\
\hline$A C T B$ & NM_173979 & GACATCCGCAAGGACСТCTA & ACATCTGCTGGAAGGTGGAC & 205 & Canel et al. 2017 \\
\hline SMARCA4 & NM_001105614.1 & CTGCAGGAACGGGAATACAG & CTGGAAGTTCAGCAGTCTGAG & 140 & This work \\
\hline
\end{tabular}

bp, basepair.

(T-9284) in DPBS for 15 min. Non-specific immunoreactions were blocked by incubation for $1 \mathrm{~h}$ in blocking solution (DPBS containing 4\% BSA and 0.2\% Tween ${ }^{\circ} 20 ;$ H5152, Promega). Incubation with Rabbit Anti-HA tag antibody (9110 Abcam) diluted 1:300 in blocking solution, was performed overnight at $4^{\circ} \mathrm{C}$. Incubation with the secondary antibody (Goat anti-Rabbit IgG, Invitrogen) was performed for $1 \mathrm{~h}$ in the dark, at room temperature. Then, embryos were mounted on glass slides, in $70 \% \mathrm{v} / \mathrm{v}$ glycerol under a cover slip using VECTASHIELD ${ }^{\circledR}$ Mounting Medium ( $\mathrm{H}-1000$, Vector Laboratories). Negative controls were generated using only the secondary antibody. For blastocyst staining, embryos were collected 7 days after IVF and ICC was performed as indicated before. To estimate TE proportion in blastocysts, two biological replicates were performed using a ready-to-use Anti-CDX2 antibody (AM392-5M, BioGenex). CDX2+ nuclei served as TE indicator, while DAPI staining was used for total nuclei count. The cell counter plugin in FIJI (ImageJ) was used for nuclei counting. Non-injected IVF blastocyst were included as control.

\section{Statistical analysis}

Differences on rates of in vitro embryo development were determined by Fisher's exact test with a $\mathrm{Cl}$ of $95 \%$, using the GraphPad Prism, version 5.01 program. Differences were considered statistically significant with a value of $P \leq 0.05$. To avoid misinterpretation due to culture procedure data was also analyzed by ANOVA, considering each biological replicate as a variable. No differences were found between replicates. For qPCR experiments, the statistical analysis was performed using GraphPad prism software version 5.01, using the MannWhitney test, with a $\mathrm{Cl}$ of $95 \%$. For each time point assessed, differences between non-injected control and injected groups were considered statistically significant with a value of $P \leq 0.05$. For mRNA decay, one biological replicate at day 7 was used as reference, to highlight the high variability within the group. For TE nuclei proportion determination one way ANOVA was used and the level of significance was $P<0.05$.

\section{Results}

\section{dCas9VP160 mRNA decay and translation during bovine preimplantation embryo development}

First, the dCas9VP160 mRNA stability and its translation into protein were evaluated during in vitro embryo development. With this aim, in vitro produced zygotes were cytoplasmically microinjected with dCas9VP160 mRNA, and thereafter, mRNA abundance was assessed by RT-qPCR during embryo development. In early embryos, transcriptional activity is limited until major embryonic genome activation, which occurs at the 8 -cell stage in bovine embryos, around day 4 of in vitro culture (Barnes \& First 1991). For this reason, to ensure CRISPR-on components availability immediately after microinjection, sgRNAs and mRNA coding for dCas9VP160 were in vitro synthesized and used for embryo microinjection.

Microinjection of mRNA had no detrimental effect on embryo development and dCas9VP160 transcripts were detected at all preimplantation stages evaluated after microinjection (Fig. 2A). As expected, dCas9VP160 mRNA abundance was significantly lower at the blastocyst stage relative to days 2 and 4 ( $p<0.05$, Fig. 2A). Furthermore, the correct translation and localization of dCas9VP160 protein was confirmed by immunostaining. dCas9VP160 protein showed nuclear localization (Fig. 2B) due to the presence of a NLS at the C-terminal of its amino acid sequence. Consistent with results for mRNA decay, dCas9VP160 protein was not detected at the blastocyst stage (Supplementary Fig. 1 , see section on supplementary materials given at the end of this article). Our results show that dCas9VP160 mRNA abundance is maintained through day 2 and 4 after injection but not at day 7 , and mRNA is efficiently translated to protein.

\section{Evaluation of the CRISPR/dCas9VP160 system for SMARCA4 and TFAP2C expression modulation in bovine embryos}

Once we verified that the components of the CRISPR-on system were efficiently delivered and dCas9VP160 was effectively translated into protein in early embryos, we next evaluated the suitability of the system for modulation of endogenous expression of SMARCA4 or TFAP2C in bovine preimplantation embryos. Figure $1 \mathrm{~A}$ illustrates the experimental design.

Presumptive zygotes were cytoplasmically injected with a mix containing dCas9VP160 mRNA and a pool 
A

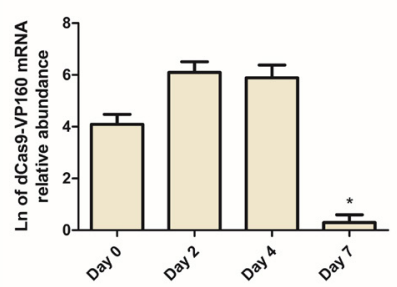

B
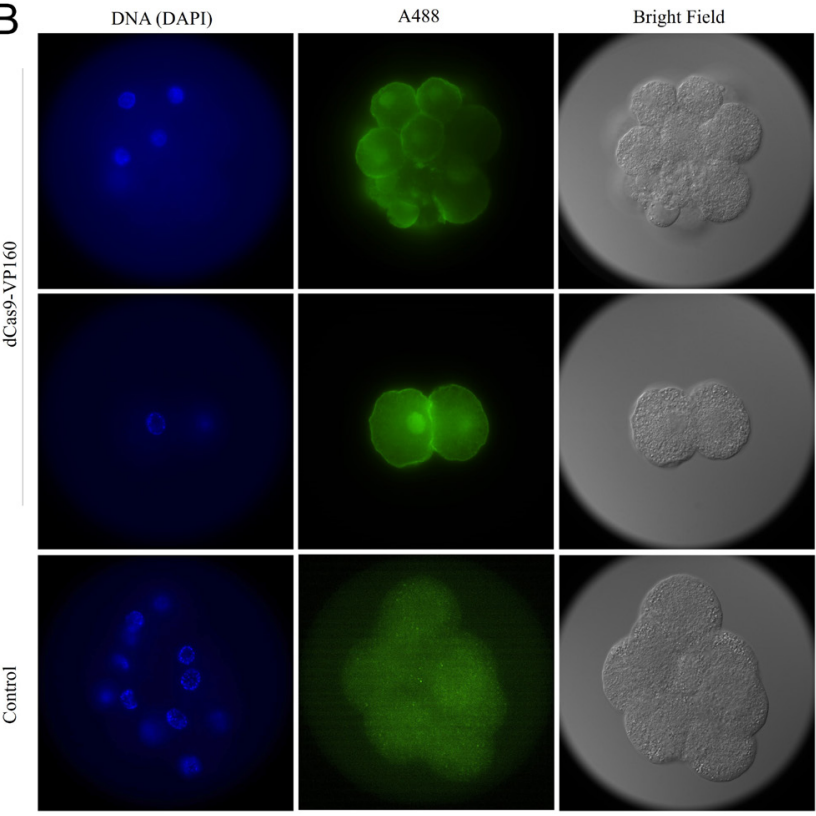

Figure 2 (A) Decay of the dCas9VP160 mRNA during in vitro development. Data is presented as the In fold-change value in mRNA abundance for each time point, relative to the amount present at blastocyst stage and normalized to the geometrical mean of Actin and GAPDH. (B) Immunodetection of dCas9VP160 protein in bovine D2 and D4 microinjected embryos. dCas9VP160 protein was detected in all blastomeric nuclei from microinjected embryos at day 2 and 4 of the in vitro culture using an anti-HA tag antibody.

of 4 sgRNAs targeting either SMARCA4 (dCas9_SM group) or TFAP2C (dCas9_TF group). The induction and kinetics of CRISPR-on endogenous gene activation were measured by RT-qPCR on days 2, 4 and 7 in pools of embryos. The expression levels of the endogenous target genes were compared to the non-injected control group.

SMARCA4 and TFAP2C transcripts were detected at all developmental stages for injected and non-injected groups. Microinjection of CRISPR-on components into one-cell stage embryos triggered a significant increase in SMARCA4 relative expression at day 2, for the dCas9_SM group. Moreover, SMARCA4 induction resulted in increased gene expression of TFAP2C and other downstream effectors of TE differentiation, such as CDX2. This result demonstrates that CRISPR-on can efficiently induce endogenous gene expression in early bovine embryos. However, no significant changes were observed in the endogenous level of NANOG. In all cases, gene expression was activated in a transient fashion, because by day 4 SMARCA4 expression levels were no longer significantly different relative to the control group (Fig. 3). Regarding dCas9_TF group, CRISPR-on microinjection did not result in statistically significant induction of TFAP2C. Nonetheless, the increase in TFAP2C was biologically relevant as a significant increase in CDX2 mRNA abundance was detected on day 2 after injection, similar to that observed for dCas9_SM group. On day 4 after injection CDX2 expression levels were no longer statistically significant. Altogether, these results demonstrate that CRISPR-on is suitable for transient induction of SMARCA4 as well as activation of downstream transcriptional targets such as CDX2 and TFAP2C.

\section{Effect of CRISPR/dCas9VP160 mediated SMARCA4 and TFAP2C expression modulation on bovine embryo development}

Next, we evaluated the effect of CRISPR-mediated temporal induction of SMARCA4 on in vitro embryo development. Although TFAP2C expression was not significantly induced, it did result in a significant induction of $C D X 2$; therefore, we also decided to assess in vitro embryo development of this group. As before, zygotes were cytoplasmically injected with a mix containing dCas9VP160 mRNA and 4 sgRNAs targeting the regulatory region of either SMARCA4 or TFAP2C and in vitro cultured until blastocyst stage. As control (SHAM), a group injected only with dCas9VP160 was included. Results of embryo development are summarized in Table 4. Interestingly, while SMARCA4 mRNA levels were increased by day 2, embryo cleavage rates were not statistically different from the control group. Instead, an effect of dCas9VP160-mediated induction of SMARCA4 expression was evident by the morulae stage, where a significantly higher rate was detected for dCas9_SM group respect to both, dCas9_TF group and dCas9VP160 control group. However, this effect was no longer noticeable by the blastocyst stage, where no significant differences were observed between microinjected groups. On the other hand, CRISPRmediated induction of TFAP $2 C$, under the conditions tested, did not affect in vitro embryo development.

Furthermore, we evaluated whether the modulation of gene expression affected the proportion of TE cells in blastocyst at day 7 after IVF. TE cells were differentially immunostained using Anti-CDX2 antibody, while total cell nuclei were labeled using DAPI. Representative examples of differential cell staining are shown in Supplementary Fig. 2A. There was no effect of gene expression modulation on the proportion of CDX2 ${ }^{+}$ blastomeres in the microinjected embryos, relative to the non-injected control (Supplementary Fig. 2B).

Together, our results suggest that CRISPR-on system had no detrimental effect on embryo development. SMARCA4 induction of expression early in the 

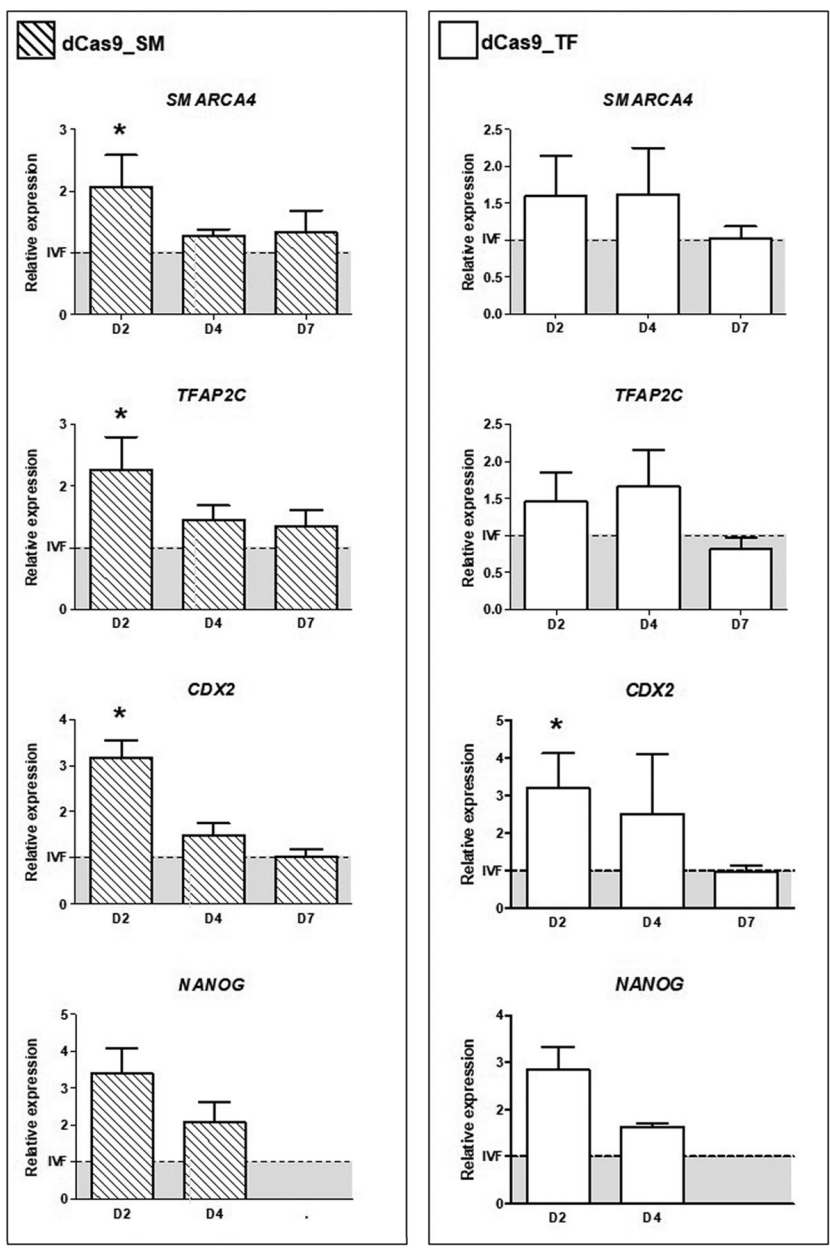

Figure 3 Relative abundance of transcripts in microinjected and control bovine embryos. The relative abundance of SMARCA4, TFAP2C, NANOG and CDX2 in embryos of the dCas9_SM group (left, striped bars) and dCas9_TF (right, white bars) was evaluated on days 2,4 and 7 of the in vitro culture. The results are expressed as fold change over IVF control embryos (dotted line). *Significant differences with respect to the IVF control, Mann Whitney $t$-test $P<0.05$.

development improved morulae rates, nonetheless blastocyst rate was not altered.

\section{Discussion}

While the CRISPR/Cas9 nuclease system has been shown to be highly efficient for genome editing in embryos of several mammalian species (Mashiko et al. 2014, Shao et al. 2014, Chen et al. 2015, Crispo et al. 2015, Wang et al. 2015a,b, Bevacqua et al. 2016), the application of the CRISPR-on system has thus far been limited to in vitro somatic cell cultures. The present work demonstrates for the first time the effectiveness of the CRISPR-on system for activation of endogenous gene expression in preimplantation bovine embryos, by simple cytoplasmic injection of RNA CRISPR components. In particular, we showed that our strategy leads to efficient delivery and
Table 4 In vitro development of bovine embryos microinjected with the CRISPR/dCas9VP160 system.

\begin{tabular}{lrrrc}
\hline Groups & $\boldsymbol{n}$ & Cleavage & Morulae & Blastocyst $(\%)$ \\
\hline dCas9_SM & 186 & $172(92)^{\mathrm{a}}$ & $115(62)^{\mathrm{a}}$ & $94(50.5)^{\mathrm{a}}$ \\
dCas9_TF & 172 & $155(90)^{\mathrm{a}}$ & $93(54)^{\mathrm{b}}$ & $70(40.7)^{\mathrm{a}}$ \\
SHAM & 62 & $58(97)^{\mathrm{a}}$ & $36(58)^{\mathrm{b}}$ & $33(53.2)^{\mathrm{a}}$ \\
\hline
\end{tabular}

$n$ : number of microinjected presumptive embryos that were in vitro cultured. Data from at least three independent replicates.

${ }^{a, b}$ Different superscripts indicate significant differences (Fisher test; $P<0.05)$.

proper translation of dCas9VP160 mRNA into protein in bovine zygotes. Moreover, dCas9VP160 triggered not only activation of the target gene shortly after microinjection, but also downstream genes. Finally, we confirmed that the CRISPR-on system is suitable for modulation of expression in early preimplantation embryos, while not causing a detrimental effect on development.

In bovine, major EGA takes place at the eightcell stage (around day four of the IVC, Barnes \& First 1991) and therefore, microinjection of CRISPR-on RNA components is required to ensure prompt availability of dCas9VP160 protein immediately after injection. In this regard, in the first experiment we assessed stability of dCas9VP160 mRNA and confirmed that it was correctly processed by the embryo translation machinery. We found that while high levels of mRNA and protein were present both at days 2 and 4; they were dramatically reduced by day 7 , probably due to mRNA and protein degradation and dilution due to cell growth and division by the blastocyst stage (Momose et al. 1999). It is interesting to note that delivery of CRISPR-on RNA components did not impair embryo development. Therefore, CRISPR-on mediated by mRNA injection constitutes an excellent system for transient activation of endogenous gene expression soon after microinjection of CRISPR-on components.

Once we confirmed that our microinjection strategy ensured proper delivery of CRISPR components and stable translation of dCas9VP160 protein, we evaluated the feasibility of employing this technology for upregulation of endogenous gene expression in embryos. While there is one report on the use of CRISPR-on system in mouse embryos (Cheng et al. 2013), it describes the transcriptional activation of a naked episomal reporter gene, which is facilitated by the presence of high number of copies of the reporter gene and avoids the complex natural genomic context of the cell. Williams et al. (2018) have reported the successful induction of endogenous gene expression in chick embryos by employing constitutively expression of CRISPR-on components. In this work, we evaluated whether endogenous gene-specific transcriptional activation can be achieved in preimplantation bovine embryos by using RNA CRISPR-on tools. As this approach is independent of gene edition (i.e., transgenesis), this 
strategy could be easily translated to bovine in vitro embryo production programs.

As a proof of principle, we employed the CRISPR-on system for upregulation of endogenous SMARCA4 and TFAP2C expression, key transcriptional regulators implicated in trophoblast lineage commitment. Previously, it was demonstrated that SMARCA4deficient blastomeres exhibit a preferential commitment to the ICM lineage (Carey et al. 2015), while TFAP2C overexpression promotes trophoblast differentiation in mouse ESCs (Kuckenberg et al. 2010). Thus, CRISPRmediated transcriptional activation of these or other TF may provide unique opportunities for understanding cell fate differentiation, with implications for in vitro embryo production improvement. Furthermore, the temporal expression pattern of SMARCA4 has not previously been studied in preimplantation bovine embryos, and there is only one report describing the expression profile of TFAP2C in bovine embryos produced by IVF (Aston et al. 2009). While not the main objective of the current study, future experiments will address whether CRISPRbased gene activation results in the modulation of cellfate decisions toward ICM or TE.

Consistent with its presumptive role in EGA, and in accordance with the mouse model, SMARCA4 transcripts were detected at all preimplantation stages in bovine embryos (Bultman et al. 2006). Notably, TFAP2C transcripts were also detected in embryos at days 2, 4 and 7 of IVC. These results are consistent with TFAP2C expression pattern in mouse embryos; however, they differ from those reported by Aston et al. for bovine embryos (2009). We confirmed that the CRISPR-on system is efficient to induce SMARCA4 expression only 2 days after microinjection of dCas9VP160 mRNA and the four sgRNAs targeting SMARCA4 promoter region (dCas9_ SM group). This prompt activation of gene expression suggests that CRISPR-on is capable of overriding the normal timing of genome activation. The CRISPR-on system targeting TFAP2C showed only a mild trend to increase TFAP2C mRNA levels (dCas9_TF group). This difference may be due to the targeted sequence itself or to inherent properties of the sgRNAs used, as it is well known that both traits critically affect CRISPR efficiency (Chakraborty et al. 2014). While achieving a mild induction of expression from the endogenous promoter is desirable for many applications, some purposes might require longer term activation of gene expression than the one reported here. In somatic cells, it was possible to induce the activation of endogenous gene expression for up to 21 days by using covalently close circle DNA (Giménez et al. 2016). Future studies will need to address whether this system can be efficiently adapted to embryos.

Importantly, the CRISPR-on system not only resulted in the modulation of its target genes, but also activation of downstream effectors known to play a role in TE maintenance. The observed overexpression of TFAP2C as consequence of SMARCA4 induction is worth mentioning, since it suggests that both of these TFs act synergistically not only in mice, but also in bovine. The interaction between SMARCA4 and TFAP2C has been reported previously in murine trophoblastic stem cells culture. The authors showed that TFAP2C and SMARCA4 bind simultaneously to the promoter region of more than 600 genes, which suggests a coordinated regulation between these transcription factors (Kidder \& Palmer 2010). In addition, CDX2, hallmark modulator of TE specification (Aston et al. 2010, Choi et al. 2012, Goissis \& Cibelli 2014), showed increased expression levels on day 2 for both, dCas9_SM and dCas9_TF groups $(P<0.05)$. Jedrusik et al. (2008) reported that CDX2 overexpression in one blastomere from a 2-cell mouse embryo triggers its commitment to the TE lineage. Therefore, the CRISPR-on system may be useful not only for in vitro reprogramming of mouse somatic cells toward iPSC (Liu et al. 2018) or trophoblast stem cells (Wei et al. 2016), but also to arbitrarily manage blastomere commitment in preimplantation embryos toward TE lineage. Further work is needed to optimize the efficiency of CRISPR-on for blastomere reprogramming.

Our data differs from expectations based on mouse reports, since NANOG expression was not repressed by SMARCA4 overexpression. Possibly, increased levels of NANOG and other TFs acting opposite to SMARCA4 and TCFAP2C were triggered secondarily to SMARCA4 and CDX2 overexpression, which could also account for the lack of CRISPR-on effect observed by day 4 . However, it is worth noting that our results are consistent with previous findings in bovine where NANOG expression is independent from CDX2. Several studies reported that bovine CDX2-defficient embryos exhibit normal NANOG expression during preimplantation development (Berg et al. 2011, Goissis \& Cibelli 2014, Wu et al. 2016), whereas in mice NANOG and CDX2 cross-regulate each other in the developing embryo (Chen et al. 2009). Our results, indeed, emphasize the species-specific differences between cattle and mouse during preimplantation development. Thus, the CRISPR-on system could help to better understand the bovine-specific early preimplantation embryonic development.

In our approach, dCas9VP160 was employed as gene activator for precise targeting of the endogenous regulatory regions of SMARCA4 and TFAP2C genes. The first version of CRISPR-based transcriptional activator or CRISPR-on, consisted on the fusion of the dCas9 protein to 4 tandem repeats of VP16 herpes virus activation domain to generate the dCas9VP64 programmable activator (Gilbert et al. 2013, Perez-Pinera et al. 2013). In mammals, VP64 recruits basal transcription machinery elements to the target promoter thus inducing gene expression (Mittler et al. 2003). However, dCas9VP64 system could only achieve moderate transcriptional activation unless multiple non-overlapping guides were 
used to synergistically target the same promoter (Cheng et al. 2013, Maeder et al. 2013, Mali et al. 2013, PerezPinera et al. 2013, Hu et al. 2014). With the fusion of 10 or 12 VP16 tandem repeats to dCas9, resulting in dCas9VP160 and dCas9VP192 respectively, the number of sgRNAs needed to induce gene expression was significantly reduced (Cheng et al. 2013, Balboa et al. 2015, reviewed by Vora et al. 2016). In its different versions, CRISPR-on technology provides a feasible approach to induce the expression of coding, non-coding, but also of extremely large genes as well as CG-rich genes for which ORF overexpression is troublesome and time consuming. Moreover, because endogenous expression is induced, natural ratios of different protein isoforms are maintained, opposite as ectopic overexpression assays (Liu et al. 2001, reviewed by La Russa \& Qi 2015. A main advantage of CRISPR-on technology is that simultaneous activation of multiple genes can be successfully achieved by using the same dCas9-based transcription factor and, furthermore, these tools are easily transferable to different species (Cheng et al. 2013).

New versions of CRISPR-on based their design on the combined use of different activation domains and also trans-recruitment strategies (reviewed by Vora et al. 2016), achieving an induction up to 300 times stronger than dCas9VP64, known as the standard activator (Tanenbaum et al. 2014, Chavez et al. 2016; reviewed by Vora et al. 2016). However, considering that in cattle blastocyst, CDX2 mRNA is enriched in TE cells by less than 2.4-fold relative to the ICM cells, we presume that dCas9VP160 mediated induction of expression would allow as to more similarly resembling endogenous gene expression as opposed to other CRISPR-on technologies, thus preventing the overload of translational resources or a toxic effect due to stoichiometric imbalance in the embryo (Moriya 2015). Lastly, the need of multiple sgRNA to target a small activation window in a specific locus, together with the mild increase in gene expression induced by dCas9VP160 activator reduces significantly the risk of undesired off target activation (Vora et al. 2016).

Finally, we evaluated the effect of the CRISPR-on system on in vitro embryo development. Interestingly, while neither cleavage nor blastocyst rates were affected, there was an improvement in development to the morula stage in the CRISPR-on SMARCA4 group. This effect might be a consequence of increased expression of SMARCA4, TFAP2C and CDX2 on day 2 of development. Because the CRISPR-on machinery was no longer active by day 7 (experiment 1), and gene expression was already compensated by that stage (experiment 2), it is not surprising that blastocysts rates or TE nuclei proportion were not affected. Although the in vitro production of blastocysts was not increased in this work, the ability of microinjected embryos to implant and survive after embryo transfer may be improved, as it was reported for other strategies including DKK1 treatment (an antagonist of the WNT signaling pathway, shown to direct lineage commitment, Denicol et al. 2014) or embryo aggregation (Misica-Turner et al. 2007).

Overall, the application of the CRISPR-on system for endogenous gene modulation of early preimplantation embryos is, to our knowledge, unprecedented and could be adapted not only to study mechanisms regulating early cell fate differentiation, but also to improve the outcome of in vitro derived embryos, by means of timerestricted overexpression of desired transcription factors.

\section{Concluding remarks}

In summary, the present study reports the use of the CRISPR-on system to induce endogenous gene expression in preimplantation bovine embryos, by simple cytoplasmic injection of RNA CRISPR components. dCas9VP160 is efficiently delivered to bovine zygotes and accurately translated into protein, triggering not only target gene expression activation shortly after microinjection, but also other TE-related genes. CRISPR-on system is suitable for its use in embryo preimplantation stages, as it does not cause a detrimental effect on development.

In conclusion, our results demonstrate that the CRISPR-on system can be used to modulate endogenous gene expression in cattle preimplantation embryos. This system may serve as a potential strategy to study early cell-fate decisions and improve TE development in preimplantation embryos.

\section{Supplementary materials}

This is linked to the online version of the paper at https://doi. org/10.1530/REP-19-0517.

\section{Declaration of interest}

Jason Knott is on the editorial board of Reproduction. Jason Knott was not involved in the review or editorial process for this paper, on which he is listed as an author. The other authors have nothing to disclose.

\section{Funding}

This work was supported by the ANPCyT from the Ministry of Science and Technology of Argentina (PICT-2018-04632).

\section{Author contribution statement}

V S and R J B took part in conceptualization and study design, performed experiments, data analysis and manuscript writing and editing. V A, N C and L R performed experiments. M I G was involved in RNA synthesis and critical discussions. R F M and D F S took part in conceptualization and study design, 
data analysis, critical discussion and manuscript drafting and critical revision. D F S and S F were in charge of financial support and resources provision. J G K was involved in study design and manuscript revision. D F S was in charge of research coordination, supervision and manuscript submission. All the authors revised and accepted the draft version of the manuscript. R J B and D F S are equal authors.

\section{Acknowledgments}

The authors sincerely thank Eco Carnes S.A. (Argentina) for providing the biological material and Maimónides University for providing the lab facilities. We would also thank Dr Oscar Taboga (CICVyA, INTA), Dr Susana Rulli (IByME-CONICET) and Catherine Wilson (MSU, USA) for their support. In addition, CONICET and Fulbright are gratefully acknowledged.

\section{References}

Aston KI, Li GP, Hicks BA, Winger QA \& White KL 2009 Genetic reprogramming of transcription factor ap-2gamma in bovine somatic cell nuclear transfer preimplantation embryos and placentomes. Cloning and Stem Cells 11 177-186. (https://doi.org/10.1089/clo.2008.0055)

Aston KI, Li GP, Hicks BA, Sessions BR, Davis AP, Rickords LF, Stevens JR \& White KL 2010 Abnormal levels of transcript abundance of developmentally important genes in various stages of preimplantation bovine somatic cell nuclear transferembryos. Cellular Reprogramming 12 23-32. (https://doi.org/10.1089/cell.2009.0042)

Avilion AA, Nicolis SK, Pevny LH, Perez L, Vivian N \& Lovell-Badge $R$ 2003 Multipotent cell lineages in early mouse development depend on SOX2 function. Genes and Development 17 126-140. (https://doi. org/10.1101/gad.224503)

Balboa D, Weltner J, Eurola S, Trokovic R, Wartiovaara K \& Otonkoski T 2015 Conditionally stabilized dCas9 activator for controlling gene expression in human cell reprogramming and differentiation. Stem Cell Reports 5 448-459. (https://doi.org/10.1016/j.stemcr.2015.08.001)

Barnes FL \& First NL 1991 Embryonic transcription in in vitro cultured bovine embryos. Molecular Reproduction and Development 29 117-123. (https://doi.org/10.1002/mrd.1080290205)

Berg DK, Smith CS, Pearton DJ, Wells DN, Broadhurst R, Donnison M \& Pfeffer PL 2011 Trophectoderm lineage determination in cattle. Developmental Cell 20 244-255. (https://doi.org/10.1016/j. devcel.2011.01.003)

Bevacqua RJ, Fernandez-Martín R, Savy V, Canel NG, Gismondi MI, Kues WA, Carlson DF, Fahrenkrug SC, Niemann H, Taboga OA et al. 2016 Efficient edition of the bovine PRNP prion gene in somatic cells and IVF embryos using the CRISPR/Cas9 system. Theriogenology 861886. e1-1896.e1. (https://doi.org/10.1016/j.theriogenology.2016.06.010)

Brackett BG \& Oliphant G 1975 Capacitation of rabbit spermatozoa in vitro. Biology of Reproduction 12 260-274. (https://doi.org/10.1095/ biolreprod12.2.260)

Bultman SJ, Gebuhr TC, Pan H, Svoboda P, Schultz RM \& Magnuson T 2006 Maternal BRG1 regulates zygotic genome activation in the mouse. Genes and Development 20 1744-1754. (https://doi.org/10.1101/ gad.1435106)

Canel NG, Bevacqua RJ, Hiriart MI, Rabelo NC, de Almeida Camargo LS, Romanato M, de Calvo LP \& Salamone DF 2017 Sperm pretreatment with heparin and I-glutathione, sex-sorting, and double cryopreservation to improve intracytoplasmic sperm injection in bovine. Theriogenology 93 62-70. (https://doi.org/10.1016/j.theriogenology.2016.12.018)

Cao Z, Carey TS, Ganguly A, Wilson CA, Paul S \& Knott JG 2015 Transcription factor AP- $2 \gamma$ induces early $\mathrm{Cd} \times 2$ expression and represses HIPPO signaling to specify the trophectoderm lineage. Development 142 1606-1615. (https://doi.org/10.1242/dev.120238)

Carey TS, Cao Z, Choi I, Ganguly A, Wilson CA, Paul S \& Knott JG 2015 BRG1 governs Nanog transcription in early mouse embryos and embryonic stem cells via antagonism of histone H3 lysine 9/14 acetylation. Molecular and Cellular Biology 35 4158-4169. (https://doi. org/10.1128/MCB.00546-15)

Chakraborty S, Ji H, Kabadi AM, Gersbach CA, Christoforou N \& Leong KW 2014 A CRISPR/Cas9-based system for reprogramming cell lineage specification. Stem Cell Reports 3 940-947. (https://doi. org/10.1016/j.stemcr.2014.09.013)

Chavatte-Palmer P, Camous S, Jammes H, Le Cleac'h N, Guillomot M \& Lee RS 2012 Review: Placental perturbations induce the developmental abnormalities often observed in bovine somatic cell nuclear transfer. Placenta 33 (Supplement) S99-S104. (https://doi.org/10.1016/j. placenta.2011.09.012)

Chavez A, Tuttle M, Pruitt BW, Ewen-Campen B, Chari R, Ter-Ovanesyan D, Haque SJ, Cecchi RJ, Kowal EJK, Buchthal J et al. 2016 Comparison of Cas9 activators in multiple species. Nature Methods 13 563-567. (https://doi.org/10.1038/nmeth.3871)

Chen L, Yabuuchi A, Eminli S, Takeuchi A, Lu CW, Hochedlinger K \& Daley GQ 2009 Cross-regulation of the Nanog and Cdx2 promoters. Cell Research 19 1052-1061. (https://doi.org/10.1038/cr.2009.79)

Chen Y, Zheng Y, Kang Y, Yang W, Niu Y, Guo X, Tu Z, Si C, Wang H, Xing R et al. 2015 Functional disruption of the dystrophin gene in rhesus monkey using CRISPR/Cas9. Human Molecular Genetics 24 3764-3774. (https://doi.org/10.1093/hmg/ddv120)

Cheng AW, Wang H, Yang H, Shi L, Katz Y, Theunissen TW, Rangarajan S, Shivalila CS, Dadon DB \& Jaenisch R 2013 Multiplexed activation of endogenous genes by CRISPR-on, an RNA-guided transcriptional activator system. Cell Research 23 1163-1171. (https://doi.org/10.1038/ cr.2013.122)

Choi I, Carey TS, Wilson CA \& Knott JG 2012 Transcription factor AP2gamma is a core regulator of tight junction biogenesis and cavity formation during mouse early embryogenesis. Development 139 46234632.

Choi I, Carey TS, Wilson CA \& Knott JG 2013 Evidence that transcription factor AP-2gamma is not required for Oct4 repression in mouse blastocysts. PLOS ONE 8 e65771. (https://doi.org/10.1371/journal. pone.0065771)

Cong L, Ran FA, Cox D, Lin S, Barretto R, Habib N, Hsu PD, Wu X, Jiang W, Marraffini LA et al. 2013 Multiplex genome engineering using CRISPR/Cas systems. Science 339 819-823. (https://doi.org/10.1126/ science.1231143)

Crispo M, Mulet AP, Tesson L, Barrera N, Cuadro F, dos Santos-Neto PC, Nguyen TH, Crénéguy A, Brusselle L, Anegón I et al. 2015 Efficient generation of myostatin knock-out sheep using CRISPR/Cas9 technology and microinjection into zygotes. PLOS ONE 10 e0136690. (https://doi. org/10.1371/journal.pone.0136690)

Degrelle SA, Campion E, Cabau C, Piumi F, Reinaud P, Richard C, Renard JP \& Hue I 2005 Molecular evidence for a critical period in mural trophoblast development in bovine blastocysts. Developmental Biology 288 448-460. (https://doi.org/10.1016/j.ydbio.2005.09.043)

Denicol AC, Block J, Kelley DE, Pohler KG, Dobbs KB, Mortensen CJ, Ortega MS \& Hansen PJ 2014 The WNT signaling antagonist Dickkopf-1 directs lineage commitment and promotes survival of the preimplantation embryo. FASEB Journal 28 3975-3986. (https://doi.org/10.1096/fj.14253112)

Engreitz J, Abudayyeh O, Gootenberg J \& Zhang F 2019 CRISPR tools for systematic studies of RNA regulation. Cold Spring Harbor Perspectives in Biology 11 a035386. (https://doi.org/10.1101/cshperspect.a035386)

Gilbert LA, Larson MH, Morsut L, Liu Z, Brar GA, Torres SE, SternGinossar N, Brandman O, Whitehead EH, Doudna JA et al. 2013 CRISPRmediated modular RNA-guided regulation of transcription in eukaryotes. Cell 154 442-451. (https://doi.org/10.1016/j.cell.2013.06.044)

Giménez CA, lelpi M, Mutto A, Grosembacher L, Argibay P \& PereyraBonnet F 2016 CRISPR-on system for the activation of the endogenous human INS gene. Gene Therapy 23 543-547. (https://doi.org/10.1038/ gt.2016.28)

Goissis MD \& Cibelli JB 2014 Functional characterization of CDX2 during bovine preimplantation development in vitro. Molecular Reproduction and Development 81 962-970. (https://doi.org/10.1002/mrd.22415)

Hill JR, Burghardt RC, Jones K, Long CR, Looney CR, Shin T, Spencer TE, Thompson JA, Winger QA \& Westhusin ME 2000 Evidence for placental abnormality as the major cause of mortality in first-trimester somatic cell cloned bovine fetuses. Biology of Reproduction 63 1787-1794. (https:// doi.org/10.1095/biolreprod63.6.1787) 
Holm P, Booth PJ, Schmidt MH, Greve T \& Callesen H 1999 High bovine blastocyst development in a static in vitro production system using SOFaa medium supplemented with sodium citrate and myo-inositol with or without serum-proteins. Theriogenology 52 683-700. (https:// doi.org/10.1016/S0093-691X(99)00162-4)

Hu J, Lei Y, Wong WK, Liu S, Lee KC, He X, You W, Zhou R, Guo JT, Chen X et al. 2014 Direct activation of human and mouse Oct4 genes using engineered TALE and Cas9 transcription factors. Nucleic Acids Research 42 4375-4390. (https://doi.org/10.1093/nar/gku109)

Jedrusik A, Parfitt DE, Guo G, Skamagki M, Grabarek JB, Johnson MH, Robson P \& Zernicka-Goetz M 2008 Role of Cdx2 and cell polarity in cell allocation and specification of trophectoderm and inner cell mass in the mouse embryo. Genes \& Development 22 2692-2706.

Jinek M, Chylinski K, Fonfara I, Hauer M, Doudna JA \& Charpentier E 2012 A programmable dual-RNA-guided DNA endonuclease in adaptive bacterial immunity. Science 337 816-821. (https://doi.org/10.1126/ science.1225829)

Jusiak B, Cleto S, Perez-Pinera P \& Lu TK 2016 Engineering synthetic gene circuits in living cells with CRISPR technology. Trends in Biotechnology 34 535-547. (https://doi.org/10.1016/j.tibtech.2015.12.014)

Khan DR, Dubé D, Gall L, Peynot N, Ruffini S, Laffont L, Le Bourhis D, Degrelle S, Jouneau A \& Duranthon V 2012 Expression of pluripotency master regulators during two key developmental transitions: EGA and early lineage specification in the bovine embryo. PLOS ONE 7 e34110. (https://doi.org/10.1371/journal.pone.0034110)

Kidder BL, Palmer S \& Knott JG 2009 SWI/SNF-Brg1 regulates selfrenewal and occupies core pluripotency-related genes in embryonic stem cells. Stem Cells 27 317-328. (https://doi.org/10.1634/stemcells.2008-0710)

Kidder BL \& Palmer S 2010 Examination of transcriptional networks reveals an important role for TCFAP2C, SMARCA4, and EOMES in trophoblast stem cell maintenance. Genome Research 20 458-472.

Knott GJ \& Doudna JA 2018 CRISPR-Cas guides the future of genetic engineering. Science 361 866-869. (https://doi.org/10.1126/science. aat5011)

Knott JG \& Paul S 2014 Transcriptional regulators of the trophoblast lineage in mammals with hemochorial placentation. Reproduction 148 R121-R136. (https://doi.org/10.1530/REP-14-0072)

Konermann S, Brigham MD, Trevino AE, Joung J, Abudayyeh OO, Barcena C, Hsu PD, Habib N, Gootenberg JS, Nishimasu H et al. 2015 Genome-scale transcriptional activation by an engineered CRISPR-Cas9 complex. Nature 517 583-588. (https://doi.org/10.1038/nature14136)

Kuckenberg P, Buhl S, Woynecki T, van Fürden B, Tolkunova E, Seiffe F, Moser M, Tomilin A, Winterhager E \& Schorle H 2010 The transcription factor TCFAP2C/AP-2gamma cooperates with CDX2 to maintain trophectoderm formation. Molecular and Cellular Biology 30 3310-3320. (https://doi.org/10.1128/MCB.01215-09)

La Russa MF \& Qi LS 2015 The new state of the art: Cas9 for gene activation and repression. Molecular and Cellular Biology 35 3800-3809. (https:// doi.org/10.1128/MCB.00512-15)

Larson MH, Gilbert LA, Wang X, Lim WA, Weissman JS \& Qi LS 2013 CRISPR interference (CRISPRi) for sequence-specific control of gene expression. Nature Protocols 8 2180-2196. (https://doi.org/10.1038/ nprot.2013.132)

Lee SH, Kwon JW, Choi I \& Kim NH 2015 Expression and function of transcriptional factor AP-2y in early embryonic development of porcine parthenotes. Reproduction, Fertility and Development 28 1197-1205. (https://doi.org/10.1071/RD14198)

Liu PQ, Rebar EJ, Zhang L, Liu Q, Jamieson AC, Liang Y, Qi H, Li PX, Chen B, Mendel MC et al. 2001 Regulation of an endogenous locus using a panel of designed zinc finger proteins targeted to accessible chromatin regions ACTIVATION of vascular endothelial growth factor A. Journal of Biological Chemistry 276 11323-11334. (https://doi. org/10.1074/jbc.M011172200)

Liu P, Chen M, Liu Y, Qi LS \& Ding S 2018 CRISPR-based chromatin remodeling of the endogenous Oct4 or Sox2 locus enables reprogramming to pluripotency. Cell Stem Cell 22 252.e4-261.e4. (https://doi.org/10.1016/j.stem.2017.12.001)

Maeder ML, Linder SJ, Cascio VM, Fu Y, Ho QH \& Joung JK 2013 Crispr RNA-guided activation of endogenous human genes. Nature Methods 10 977-979. (https://doi.org/10.1038/nmeth.2598)

Mainigi MA, Sapienza C, Butts S \& Coutifaris C 2016 A molecular perspective on procedures and outcomes with assisted reproductive technologies. Cold Spring Harbor Perspectives in Medicine 6 a023416. (https://doi.org/10.1101/cshperspect.a023416)

Mali P, Aach J, Stranges PB, Esvelt KM, Moosburner M, Kosuri S, Yang L \& Church GM 2013 CAS9 transcriptional activators for target specificity screening and paired nickases for cooperative genome engineering. Nature Biotechnology 31 833-838. (https://doi.org/10.1038/nbt.2675)

Marshall OJ 2004 PerlPrimer: cross-platform, graphical primer design for standard, bisulphite and real-time PCR. Bioinformatics 20 2471-2472. (https://doi.org/10.1093/bioinformatics/bth254)

Mashiko D, Young SA, Muto M, Kato H, Nozawa K, Ogawa M, Noda T, Kim YJ, Satouh Y, Fujihara Y et al. 2014 Feasibility for a large scale mouse mutagenesis by injecting CRISPR/Cas plasmid into zygotes. Development, Growth and Differentiation 56 122-129. (https://doi. org/10.1111/dgd.12113)

Masui S, Nakatake Y, Toyooka Y, Shimosato D, Yagi R, Takahashi K, Okochi H, Okuda A, Matoba R, Sharov AA et al. 2007 Pluripotency governed by Sox2 via regulation of Oct $3 / 4$ expression in mouse embryonic stem cells. Nature Cell Biology 9 625-635. (https://doi. org/10.1038/ncb1589)

Misica-Turner PM, Oback FC, Eichenlaub M, Wells DN \& Oback B 2007 Aggregating embryonic but not somatic nuclear transfer embryos increases cloning efficiency in cattle. Biology of Reproduction $\mathbf{7 6}$ 268-278. (https://doi.org/10.1095/biolreprod.106.050922)

Mittler G, Stühler T, Santolin L, Uhlmann T, Kremmer E, Lottspeich F, Berti L \& Meisterernst M 2003 A novel docking site on Mediator is critical for activation by VP16 in mammalian cells. EMBO Journal 22 6494-6504. (https://doi.org/10.1093/emboj/cdg619)

Mojica FJ, Díez-Villaseñor C, García-Martínez J \& Soria E 2005 Intervening sequences of regularly spaced prokaryotic repeats derive from foreign genetic elements. Journal of Molecular Evolution 60 174-182. (https:// doi.org/10.1007/s00239-004-0046-3)

Momose T, Tonegawa A, Takeuchi J, Ogawa H, Umesono K \& Yasuda K 1999 Efficient targeting of gene expression in chick embryos by microelectroporation. Development, Growth and Differentiation 41 335-344. (https://doi.org/10.1046/j.1440-169x.1999.413437.x)

Moriya H 2015 Quantitative nature of overexpression experiments. Molecular Biology of the Cell 26 3932-3939. (https://doi.org/10.1091/ mbc.E15-07-0512)

Ozawa M, Sakatani M, Yao J, Shanker S, Yu F, Yamashita R, Wakabayashi S, Nakai K, Dobbs KB, Sudano MJ et al. 2012 Global gene expression of the inner cell mass and trophectoderm of the bovine blastocyst. BMC Developmental Biology 12 33. (https://doi.org/10.1186/1471213X-12-33)

Perez-Pinera P, Kocak DD, Vockley CM, Adler AF, Kabadi AM, Polstein LR, Thakore PI, Glass KA, Ousterout DG, Leong KW et al. 2013 RNAguided gene activation by CRISPR-Cas9-based transcription factors. Nature Methods 10 973-976. (https://doi.org/10.1038/nmeth.2600)

Qi LS, Larson MH, Gilbert LA, Doudna JA, Weissman JS, Arkin AP \& Lim WA 2013 Repurposing CRISPR as an RNA-guided platform for sequencespecific control of gene expression. Cell 152 1173-1183. (https://doi.org/10.1016/j.cell.2013.02.022)

Rossant J 2004 Lineage development and polar asymmetries in the periimplantation mouse blastocyst. Seminars in Cell and Developmental Biology 15 573- 581. (https://doi.org/10.1016/j.semcdb.2004.04.003)

Shao Y, Guan Y, Wang L, Qiu Z, Liu M, Chen Y, Wu L, Li Y, Ma X, Liu M et al. 2014 CRISPR/Cas mediated genome editing in the rat via direct injection of one-cell embryos. Nature Protocols 9 2493-2512. (https:// doi.org/10.1038/nprot.2014.171)

Shen B, Zhang W, Zhang J, Zhou J, Wang J, Chen L, Wang L, Hodgkins A, lyer V, Huang X et al. 2014 Efficient genome modification by CRISPRCas9 nickase with minimal off-target effects. Nature Methods $\mathbf{1 1}$ 399-402. (https://doi.org/10.1038/nmeth.2857)

Simmet K, Zakhartchenko V, Philippou-Massier J, Blum H, Klymiuk N \& Wolf E 2018 OCT4/POU5F1 is required for NANOG expression in bovine blastocysts. PNAS 115 2770-2775. (https://doi.org/10.1073/ pnas.1718833115)

Strumpf D, Mao CA, Yamanaka Y, Ralston A, Chawengsaksophak K, Beck F \& Rossant J 2005 Cdx2 is required for correct cell fate specification and differentiation of trophectoderm in the mouse blastocyst. Development 132 2093-2102. (https://doi.org/10.1242/dev.01801)

Tanenbaum ME, Gilbert LA, Qi LS, Weissman JS \& Vale RD 2014 A protein-tagging system for signal amplification in gene expression and 
fluorescence imaging. Cell 159 635-646. (https://doi.org/10.1016/j. cell.2014.09.039)

Tripurani SK, Lee KB, Wang L, Wee G, Smith GW, Lee YS, Latham KE \& Yao J 2011 A novel functional role for the oocyte-specific transcription factor newborn ovary homeobox (NOBOX) during early embryonic development in cattle. Endocrinology 152 1013-1023. (https://doi. org/10.1210/en.2010-1134)

Vora S, Tuttle M, Cheng J \& Church G 2016 Next stop for the CRISPR revolution: RNA-guided epigenetic regulators. FEBS Journal 283 3181-3193. (https://doi.org/10.1111/febs.13768)

Wang X, Yu H, Lei A, Zhou J, Zeng W, Zhu H, Dong Z, Niu Y, Shi B, Cai B et al. 2015a Generation of gene-modified goats targeting MSTN and FGF5 via zygote injection of CRISPR/Cas9 system. Scientific Reports $\mathbf{5}$ 13878. (https://doi.org/10.1038/srep13878)

Wang Y, Du Y, Shen B, Zhou X, Li J, Liu Y, Wang J, Zhou J, Hu B, Kang N et al. $2015 \mathrm{~b}$ Efficient generation of gene-modified pigs via injection of zygote with Cas9/sgRNA. Scientific Reports 5 8256. (https://doi. org/10.1038/srep08256)

Wei S, Zou Q, Lai S, Zhang Q, Li L, Yan Q, Zhou X, Zhong H \& Lai L 2016 Conversion of embryonic stem cells into extraembryonic lineages by CRISPR-mediated activators. Scientific Reports 6 19648. (https://doi. org/10.1038/srep19648)

Williams RM, Senanayake U, Artibani M, Taylor G, Wells D, Ahmed AA \& Sauka-Spengler T 2018 Genome and epigenome engineering CRISPR toolkit for in vivo modulation of cis-regulatory interactions and gene expression in the chicken embryo. Development 145 dev160333. (https://doi.org/10.1242/dev.160333)

Winger Q, Huang J, Auman HJ, Lewandoski M \& Williams T 2006 Analysis of transcription factor AP2 expression and function during mouse preimplantation development. Biology of Reproduction 75 324-333. (https://doi.org/10.1095/biolreprod.106.052407)

Wrenzycki C, Lucas-Hahn A, Herrmann D, Lemme E, Korsawe K \& Niemann H 2002 In vitro production and nuclear transfer affect dosage compensation of the X-linked gene transcripts G6PD, PGK, and Xist in preimplantation bovine embryos. Biology of Reproduction 66 127-134. (https://doi.org/10.1095/biolreprod66.1.127)

Wu X, Song M, Yang X, Liu X, Liu K, Jiao C, Wang J, Bai C, Su G, Liu X et al. 2016 Establishment of bovine embryonic stem cells after knockdown of CDX2. Scientific Reports 6 28343. (https://doi.org/10.1038/ srep28343)

Zalatan JG, Lee ME, Almeida R, Gilbert LA, Whitehead EH, La Russa M, Tsai JC, Weissman JS, Dueber JE, Qi LS et al. 2015 Engineering complex synthetic transcriptional programs with CRISPR RNA scaffolds. Cell 160 339-350. (https://doi.org/10.1016/j.cell.2014.11.052)

Zhenhua G, Rajput SK, Folger JK, Di L, Knott JG \& Smith GW 2017 Pre- and peri-/post-compaction follistatin treatment increases in vitro production of cattle embryos. PLOS ONE 12 e0170808. (https://doi. org/10.1371/journal.pone.0170808)

Received 30 October 2019

First decision 22 November 2019

Revised manuscript received 25 March 2020

Accepted 2 April 2020 